\title{
Quantum-well states and induced magnetism in Fe/CuN/Fe bcc (001) trilayers
}

Niklasson, A.M.N.; Mirbt, S.; Skriver, Hans Lomholt; Johansson, B.

Published in:

Physical Review B

Link to article, DOI:

10.1103/PhysRevB.53.8509

Publication date:

1996

Document Version

Publisher's PDF, also known as Version of record

Link back to DTU Orbit

Citation (APA):

Niklasson, A. M. N., Mirbt, S., Skriver, H. L., \& Johansson, B. (1996). Quantum-well states and induced magnetism in Fe/CuN/Fe bcc (001) trilayers. Physical Review B, 53(13), 8509-8514.

https://doi.org/10.1103/PhysRevB.53.8509

\section{General rights}

Copyright and moral rights for the publications made accessible in the public portal are retained by the authors and/or other copyright owners and it is a condition of accessing publications that users recognise and abide by the legal requirements associated with these rights.

- Users may download and print one copy of any publication from the public portal for the purpose of private study or research.

- You may not further distribute the material or use it for any profit-making activity or commercial gain

- You may freely distribute the URL identifying the publication in the public portal 


\title{
Quantum-well states and induced magnetism in $\mathrm{Fe} / \mathrm{Cu}{ }_{N} / \mathrm{Fe}$ bcc (001) trilayers
}

\author{
A. M. N. Niklasson and S. Mirbt \\ Condensed Matter Theory Group, Physics Department Uppsala University, S-75121 Uppsala, Sweden \\ H. L. Skriver \\ Center for Atomic-scale Materials Physics and Physics Department, Technical University of Denmark, DK-2800 Lyngby, Denmark \\ B. Johansson \\ Condensed Matter Theory Group, Physics Department Uppsala University, S-75121 Uppsala, Sweden
}

(Received 22 August 1995)

\begin{abstract}
We have used a first-principles Green's function technique to investigate the formation of magnetic moments in $\mathrm{Fe} / \mathrm{Cu}_{N} / \mathrm{Fe}$ bcc (001) trilayers. We show that the magnetic moment in the paramagnetic spacer material to a first approximation may be described as a linear superposition of the magnetic profiles of two single $\mathrm{Fe} / \mathrm{Cu}$ interfaces. The small deviations from this simple superposition are shown to be a consequence of quantum-well states confined within the paramagnetic spacer. This connection is confirmed by direct calculation of the state density. The results are of conceptual interest for the understanding of the exchange coupling.
\end{abstract}

\section{INTRODUCTION}

The advance of epitaxially grown magnetic multilayer systems has created a rapidly developing field in materials physics where artificially made systems show unusual properties such as long-range oscillatory exchange interactions ${ }^{1-3}$ and "giant" magnetoresistance effects. ${ }^{4,5}$ Much experimental and theoretical work on these multilayer systems has been devoted to the study of the exchange interaction and magnetoresistance, and less attention has been devoted to the distribution of the magnetic moment in the spacer region. The present paper is an attempt to fill this gap. In particular, we discuss the formation of an oscillatory magnetic moment in the paramagnetic spacer and its relation to the exchange interaction and the formation of quantum-well (QW) states of the kind suggested by Ortega and Himpsel. ${ }^{6}$

Structures with oscillatory exchange couplings are usually produced as sandwiches or trilayers of a ferromagnetic transition metal spaced by a varying number of paramagnetic metal layers. The trilayer sandwich $\mathrm{Fe} / \mathrm{Cu}_{N} / \mathrm{Fe}$, which we have studied, consists of two semi-infinite bcc (001) crystals of ferromagnetic Fe separated by a varying number $N$ of paramagnetic $\mathrm{Cu}$ monolayers. The entire sandwich is assumed to have the same structure as the semi-infinite Fe crystals which means that lattice relaxations are neglected. Large relaxations will of course change the width of the quantum well formed between the two Fe crystals and may also affect the magnetic moment especially at the interfaces. Owing to the reconstruction of $\mathrm{Cu}$ on bcc $\mathrm{Fe}$ it is difficult to produce coherent, epitaxially grown trilayers of $\mathrm{Fe} / \mathrm{Cu} / \mathrm{Fe}$ and this system is much less studied than, for example, $\mathrm{Co} / \mathrm{Cu} / \mathrm{Co}$. However, from a theoretical point of view the $\mathrm{Fe} / \mathrm{Cu}_{N} / \mathrm{Fe}$ bcc (001) trilayer has turned out to be ideal because of a well-defined quantum well (QW) for the minority spin states in the $\mathrm{Cu}$ spacer. This is in contrast to the $\mathrm{Co} /$ $\mathrm{Cu} / \mathrm{Co}$ system structure where the reflection at the magnetic interfaces is relatively weak in the region close to the Fermi level. $^{7,8}$
To understand how the magnetic moments are formed in the trilayer system, we have also investigated a single interface of $\mathrm{Fe} / \mathrm{Cu}$ constructed of two semi-infinite bcc (001) crystals. This allows us to isolate the QW contributions to the magnetic moments in the $\mathrm{Cu}$ spacer of the trilayer and develop a simple picture of the formation of the oscillating magnetic moment profile. In addition, we show how the magnetization is connected to the QW states which give rise to the exchange coupling across the spacer material.

\section{THEORY}

In this section we show how the magnetic contribution from multiple-scattering effects such as QW states in a trilayer may be extracted by means of a superposition of the magnetic profiles of two single, freestanding interfaces.

\section{A. Multiple-scattering effects}

The ferromagnetic semi-infinite Fe crystals on the left$(L)$ and right-hand $(R)$ sides of the trilayer may be regarded as spin-dependent perturbations $V_{L}^{\sigma}$ and $V_{R}^{\sigma}$, which create a magnetic quantum well in the spacer material. These magnetic perturbations change the ground-state Green's function $G_{0}$ of the bulk paramagnet and, if we ignore self-consistency effects in the spacer layers, the perturbed Green's function: $G^{\sigma}$ may be given by a Dyson series which we choose to separate as follows

$$
G^{\sigma}=G_{0}+\Delta G_{L}^{\sigma}+\Delta G_{R}^{\sigma}+\Delta G_{\mathrm{QW}}^{\sigma} .
$$

Here $\sigma$ denotes the separate spin channels and

$$
\begin{aligned}
& \Delta G_{L}^{\sigma}=G_{0} V_{L}^{\sigma} G_{0}+G_{0} V_{L}^{\sigma} G_{0} V_{L}^{\sigma} G_{0}+\cdots, \\
& \Delta G_{R}^{\sigma}=G_{0} V_{R}^{\sigma} G_{0}+G_{0} V_{R}^{\sigma} G_{0} V_{R}^{\sigma} G_{0}+\cdots,
\end{aligned}
$$

and

$$
\Delta G_{\mathrm{QW}}^{\sigma}=G_{0} V_{L}^{\sigma} G_{0} V_{R}^{\sigma} G_{0}+\cdots
$$


In these equations $\Delta G_{L}^{\sigma}$ includes all scattering events at the left-hand potential barrier which may be recognized as a single-interface perturbation of the spacer material due to the magnetic semi-infinite crystal on the left, without any interaction with the Fe crystal on the right-hand side. Similarly, $\Delta G_{R}^{\sigma}$ is the single interface contribution associated with the perturbation on the right-hand side. In the case where the $\mathrm{Fe}$ moments are coupled ferromagnetically (FM) across the spacer, i.e., symmetric boundary conditions, we have the relation

$$
\Delta G_{R}^{\sigma}\left(r, r^{\prime}, E\right)=\Delta G_{L}^{\sigma}\left(-r,-r^{\prime}, E\right),
$$

whereas in the antiferromagnetic (AFM) case we have

$$
\Delta G_{R}^{\sigma}\left(r, r^{\prime}, E\right)=\Delta G_{L}^{-\sigma}\left(-r,-r^{\prime}, E\right),
$$

where the origin of the space coordinates $r, r^{\prime}$ is taken to be the center of the trilayer. The final mixed interaction term, $\Delta G_{\mathrm{QW}}^{\sigma}$, includes all multiple-scattering events related to the presence of both interfaces and therefore includes the effects of possible QW states confined to the quantum well formed between the two $\mathrm{Fe} / \mathrm{Cu}$ interfaces similar to standing waves in a box.

From Eq. (1) one may define a Green's function

$$
\tilde{G}^{\sigma}=G_{0}+\Delta G_{L}^{\sigma}+\Delta G_{R}^{\sigma},
$$

which does not include the QW contributions. In the paramagnetic spacer the magnetic spin density $m(r)$ is given by

$$
m(r)=-\frac{1}{\pi} \int^{E_{F}} d E \operatorname{Im}\left[G^{\uparrow}(r, r, E)-G^{\downarrow}(r, r, E)\right],
$$

and if we exclude QW contributions, i.e., use Eq. (7), the magnetic spin density $\tilde{m}(r)$ in a trilayer may be expressed as a superposition of two independent, single-interface magnetization profiles associated with the left- and the right-hand $\mathrm{Fe} / \mathrm{Cu}$ interfaces, respectively. We have

$$
\tilde{m}(r)=m_{L}(r)+m_{R}(r),
$$

where

$m_{L}(r)=-\frac{1}{\pi} \int^{E_{F}} d E \operatorname{Im}\left[\Delta G_{L}^{\uparrow}(r, r, E)-\Delta G_{L}^{\downarrow}(r, r, E)\right]$

and

$$
m_{R}(r)=( \pm) m_{L}(-r) \text {. }
$$

In Eq. (11), + and - correspond to ferromagnetic and antiferromagnetic boundary conditions, respectively.

The single freestanding interface magnetization profiles $m_{L}(r)$ and $m_{R}(r)$ are magnetic Friedel oscillations induced in the spacer and they may be calculated separately. If we therefore integrate the spin density over the atomic sphere centered at layer $n$ to obtain a layer-resolved magnetic moment and form the difference

$$
M_{\mathrm{QW}}(n)=m(n)-\tilde{m}(n),
$$

where $m(n)$ is the total magnetic moment profile of the trilayer and includes all multiple-scattering effects, we have isolated the possible QW contribution to the magnetic profile. An appreciable deviation $M_{\mathrm{QW}}(n)$ from the linear super- position may therefore serve as an indication of magnetic QW states confined in the trilayer.

We have thus shown how one may isolate possible QW contributions to the magnetic profile. This has been shown by means of the Dyson series which does not include selfconsistency effects. However, in our calulations, the magnetic profile of the trilayer, $m(n)$, as well as the magnetic Friedel oscillations, $m_{L}(n)$ and $m_{R}(n)$, are all calculated in self-consistent procedures. In this way self-consistency effects connected with the single interfaces will be included in the superposition, Eq. (9). Remaining self-consistency effects related to the formation of the quantum well are included in $M_{\mathrm{QW}}(n)$. However, these effects turn out to be small and the linear superposition is in fact a good approximation to the correct magnetization.

\section{B. Recursion relation}

If the linear superposition, Eq. (9), gives a good description of the oscillating magnetic moments in the spacer material, it is possible to predict the magnetic profile for a particular spacer thickness once it is known for two other thicknesses. From Eq. (9) one may derive the following trilayer recursion relation between three succeeding spacer thicknesses:

$$
\begin{aligned}
m^{N-1}(n)= & m^{N}(n)+m^{N}(n+1)-m^{N+1}(n+1) \\
& (n=1,2, \ldots, N-1),
\end{aligned}
$$

where $m^{N}(n)$ is the magnetic moment in the atomic monolayer $n$ for a trilayer with $N$ number of atomic interlayers. Here, the spacer layer number $n$ is counted from one interface to the other. This trilayer recursive relation is valid for FM as well as AFM boundary conditions and may also be applied to multilayer systems. It may be used to predict the behavior of the magnetic moment profile and deviations from the true profile interpreted as the multiple-scattering effects $\Delta G_{\mathrm{QW}}^{\sigma}$. In the present case it has served as a check of the validity of the linear superposition, Eq. (9).

\section{METHOD OF CALCULATION}

The present calculations were performed by means of the interface Green's function technique, developed by Skriver and Rosengaard. ${ }^{9}$ This method is based on the linear muffintin orbital (LMTO) method ${ }^{10,11}$ within the tight-binding, ${ }^{12-14}$ frozen core, and atomic-sphere approximations together with the local spin density approximation as parametrized by Vosko, Wilk, and Nusair. ${ }^{15}$ The application of a Green's function technique ensures a correct description of the loss of translational symmetry perpendicular to the interfaces. Furthermore, the atomic-sphere approximation and the application of the principle layer technique ${ }^{16}$ lead to a high computational efficiency. The method has recently been used in studies of surface magnetism and surface core level shifts, ${ }^{17-19}$ and subsequently also applied to the magnetic multilayer problem by Mirbt et al. ${ }^{20}$

To calculate the spectral density for the spacer region

$$
D^{\sigma}\left(\mathbf{k}_{\|}, E\right)=-\frac{1}{\pi} \operatorname{Im} \operatorname{Tr} G^{\sigma}\left(\mathbf{k}_{\|}, E\right)
$$




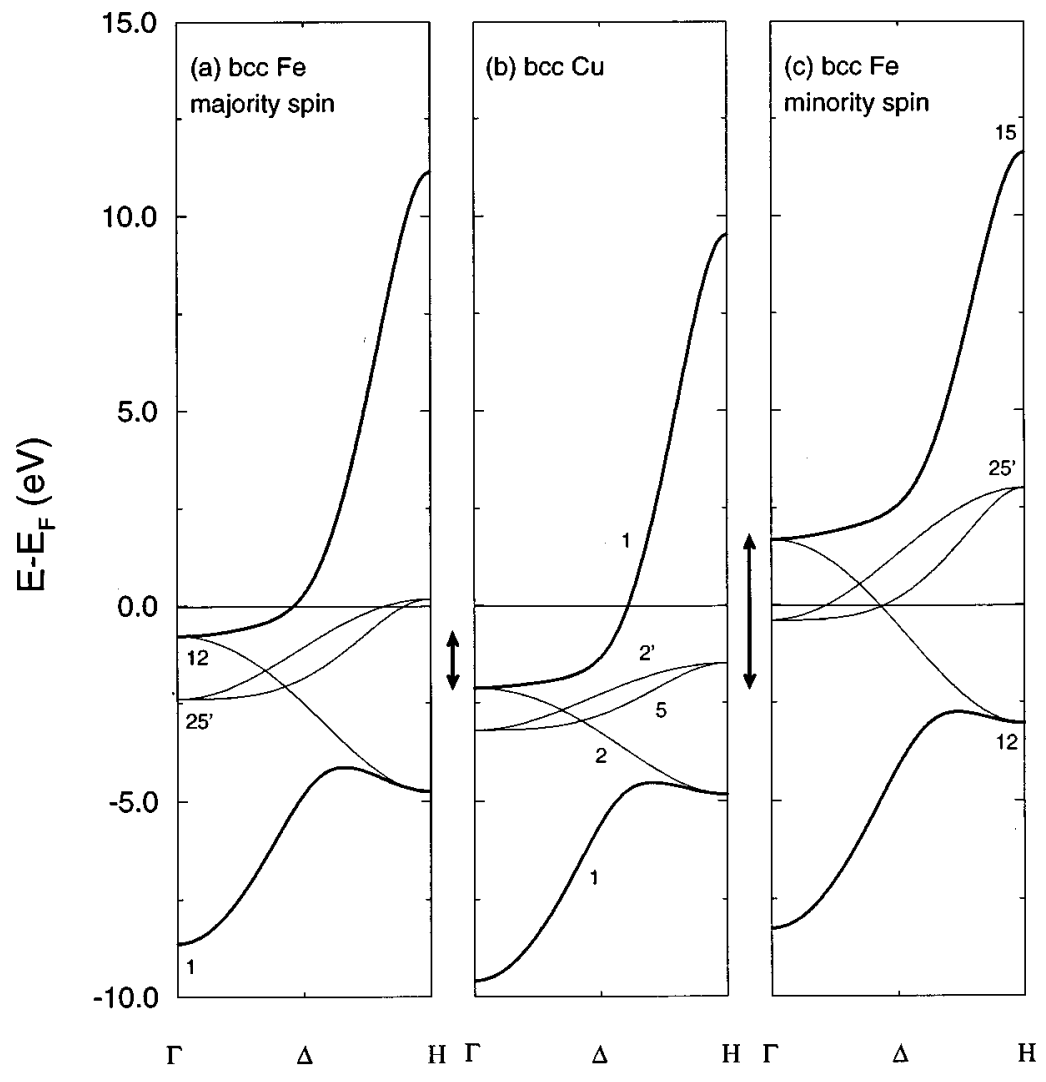

FIG. 1. The bulk energy band structure of bcc $\mathrm{Cu}$ and bcc Fe along the (001) direction. The vanishing overlap between the $s p$ bands, $\Gamma_{12}-\Delta_{1}$ $H_{15}$, in $\mathrm{Cu}$ and $\mathrm{Fe}$ creates spin-dependent gaps at the $\mathrm{Cu} / \mathrm{Fe}$ interfaces (marked with arrows) which give rise to well-defined quantum wells. from the interface Green's function of spin $\sigma$, wave vector $\mathbf{k}_{\|}$, and energy $E$ we follow a procedure originally suggested by Kudrnovsky ${ }^{21}$ for the related problem of obtaining a state density from the Green's function. First the Green's function is calculated on a linear contour below the real axis in the complex energy plane. Then the partial derivatives of the Cauchy-Riemann relations are calculated by numerical differentiation and used to estimate the Green's function on a contour closer to the real axis than the original contour. By repeating this procedure until the real axis is reached one may eventually obtain a good estimate of the spectral density. This procedure has the advantage over the straightforward technique where one performs a direct calculation of the Green's function close to the real axis that poles are not missed and that the spectral density obtained in this fashion may easily be integrated over $\mathbf{k}_{\|}$to give the local state density.

\section{QUANTUM-WELL STATES IN $\mathrm{Fe} / \mathrm{Cu}_{N} / \mathrm{Fe}$}

The formation of magnetic quantum wells in the $\mathrm{Fe} / \mathrm{Cu}_{N} / \mathrm{Fe}$ trilayer may be explained in terms of the bulk electronic structures of bcc $\mathrm{Fe}$ and $\mathrm{Cu}$ shown in Fig. 1. Of particular interest are states in the $\mathrm{Cu} s p$ band, $\Gamma_{12}-\Delta_{1}$ $H_{15}$, which because of vanishing overlap cannot scatter into the Fe majority $s p$ band in the energy range from $\Gamma_{12}(\mathrm{Cu})$ to $\Gamma_{12}(\mathrm{Fe} \uparrow)$ and into the $\mathrm{Fe}$ minority band in the energy range from $\Gamma_{12}(\mathrm{Cu})$ to $\Gamma_{12}(\mathrm{Fe} \downarrow)$. As a result, electrons with majority spin and with energies from $-2.1 \mathrm{eV}$ to $-0.8 \mathrm{eV}$ and electrons with minority spin and with energies from $-2.1 \mathrm{eV}$ to $1.7 \mathrm{eV}$ experience total reflection at the interfaces and will therefore be confined to the $\mathrm{Cu}$ spacer. In a very simplified picture this may be described as a potential box where the heights of the walls are spin dependent. Such a potential-well model exhibits many of the features of the real trilayer system, such as oscillating exchange coupling and confined QW states. $^{22,8}$

To confirm the picture of magnetic QW states confined to the paramagnetic $\mathrm{Cu}$ spacer region we have performed selfconsistent calculations of the Green's function for trilayers of $\mathrm{Fe} / \mathrm{Cu} / \mathrm{Fe}$ with a varying number of $\mathrm{Cu}$ interlayers. In Fig. 2 we show the corresponding spectral density evaluated at the center $\bar{\Gamma}$ of the 2D Brillouin zone for both spin channels with a FM coupling of the spins of the two sides of the trilayer and for a particular choice of spacer thickness. The spectral density clearly exhibits sharp resonances corresponding to standing waves in a box which are not present in bulk $\mathrm{Cu}$ nor are they seen in the case of a single $\mathrm{Fe} / \mathrm{Cu}$ interface. In addition, one observes no resonances of majority spin above $-0.8 \mathrm{eV}$, i.e., above the top of the majority-spin $\mathrm{QW}$, and no resonances of minority spin above $1.7 \mathrm{eV}$, i.e., above the top of the minority-spin QW. The sharp resonances in the spectral density may therefore be associated with magnetic QW states confined to the $\mathrm{Cu}$ spacer. In the AFM case the magnetic quantum well is asymmetric and there are only $\mathrm{QW}$ states in the narrow region below $-0.8 \mathrm{eV}$ for both spin channels. It follows that close to the Fermi level there are only QW states for FM coupling and minority spin.

In the following we shall discuss the effects of all QW states in terms of the calculated spectral density at $\bar{\Gamma}$. This is possible because the QW states exhibit a weak quadratic $k_{\|}$ dispersion as may be seen in Fig. 3 where we show the spectral density $D^{-}\left(k_{x}, k_{y}=0, E\right)$ along the $k_{x}$ axis parallel to the interfaces. As a result the maximum of the integrated 


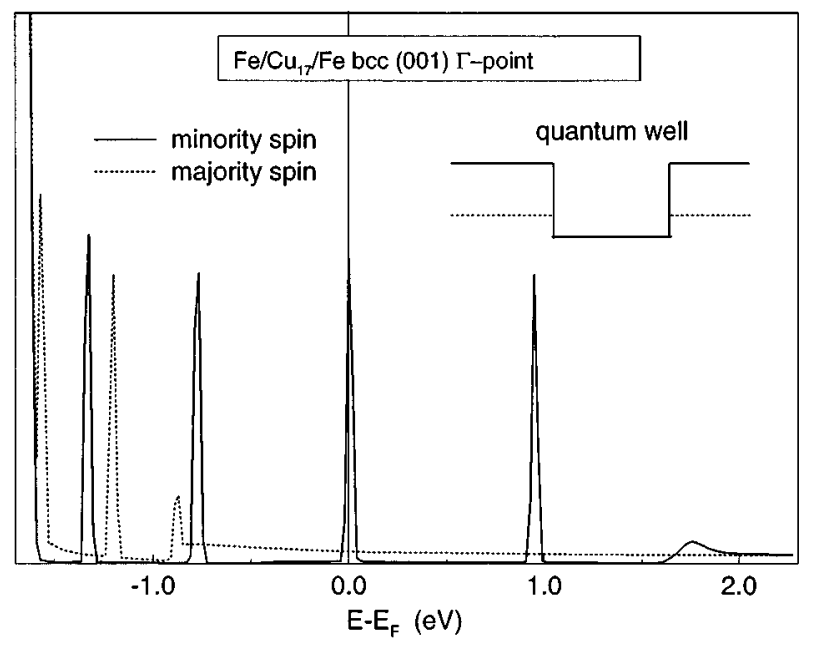

FIG. 2. The spectral density at $\bar{\Gamma}$ for a $\mathrm{Fe} / \mathrm{Cu}_{17} / \mathrm{Fe}$ bcc (001) trilayer with FM boundary condition for the majority spin (solid line) and the minority spin (dotted line). The periodic resonances in the spectral density correspond to QW states confined to the spacer layers. A quantum well, shown schematically in the upper righthand corner, gives rise to $\mathrm{QW}$ states very similar to the calculated states.

spectral density shown in the lower panel and the total state density coincides with the resonances of the spectral density at $\bar{\Gamma}$. Hence, the latter may be used to represent the QW influence on the total state density.

\section{MAGNETIC-MOMENT PROFILE}

\section{A. Friedel tail superposition}

As an example of how the superposition, Eq. (9), of independent magnetic Friedel tails from two single $\mathrm{Fe} / \mathrm{Cu}$ interfaces describes the magnetic profile in a trilayer we display

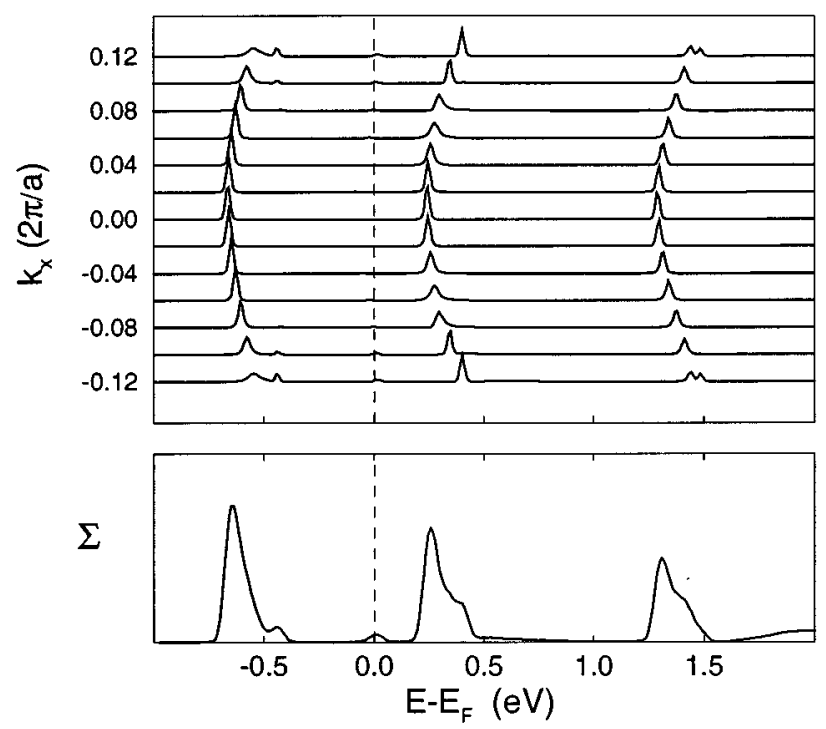

FIG. 3. The spectral density, $D^{-}\left(k_{x}, k_{y}=0, E\right)$, close to the center of the $2 \mathrm{D}$ Brillouin zone $\bar{\Gamma}$, upper panel, and the corresponding integrated state density, lower panel. $k_{x}$ is in units of $2 \pi / a$ where $a$ is the lattice spacing.

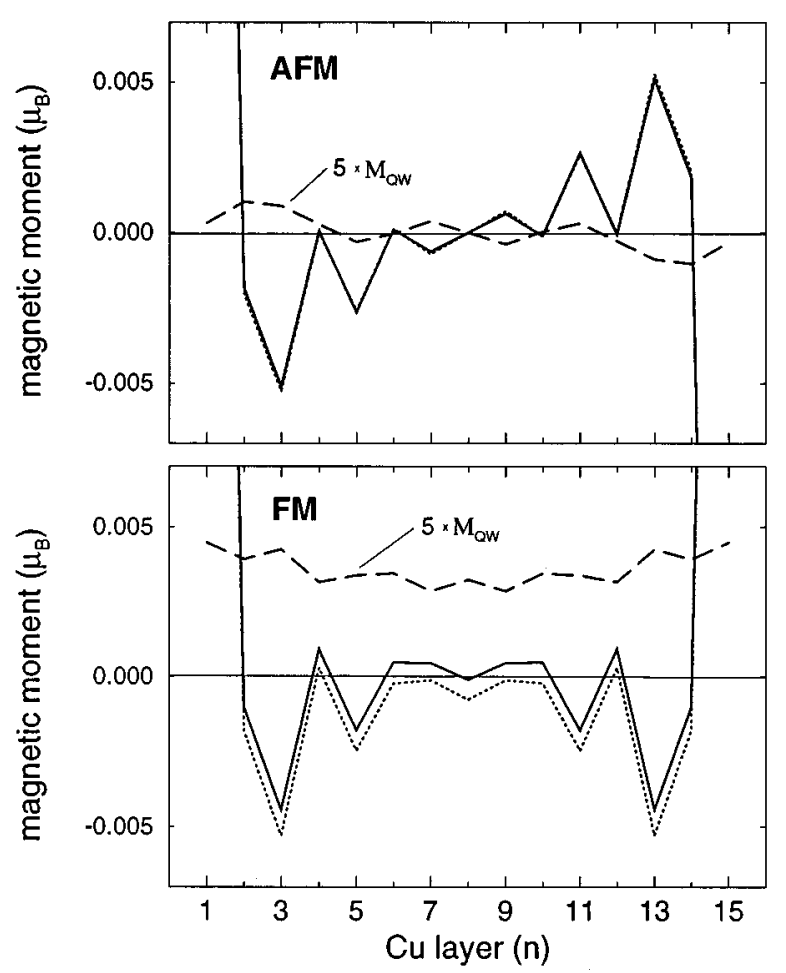

FIG. 4. Magnetic profiles in a $\mathrm{Fe} / \mathrm{Cu}_{15} / \mathrm{Fe}$ bcc (001) trilayer for ferromagnetic (FM) and antiferromagnetic (AFM) coupling. The thin solid lines are the magnetic spin moments from a selfconsistent trilayer calculation. The thin dotted lines are the magnetic moments calculated from the superposition of the magnetic profiles of two independent single $\mathrm{Fe} / \mathrm{Cu}$ interfaces, Eq. (9). The bold long-dashed lines are 5 times the difference, $M_{\mathrm{QW}}(n)$, between the self-consistent moments and superposed moments, Eq. (12).

in Fig. 4 the magnetic-moment profiles in a $\mathrm{Fe} / \mathrm{Cu}_{15} / \mathrm{Fe} \mathrm{bcc}$ (001) trilayer. In the case of AFM coupled Fe crystals, upper panel, one observes that the deviation from the linear superposition, $M_{\mathrm{QW}}(n)$ Eq. (12), is negligible, i.e., that there is essentially no difference between the superposed and the self-consistent moments profiles. This is in agreement with the absence of QW states close to the Fermi level for AFM coupling as discussed in the previous section. In the case of FM coupled Fe crystals, lower panel, one finds a $M_{\mathrm{QW}}(n)$ which is essentially constant throughout the spacer layers and comparable in magnitude to the lowest moments found at the central layers of the trilayer. This constant deviation between the correct moment profile and the superposed moment profile is a result of the presence of QW states in the minority-spin channel close to the Fermi level for FM coupling.

\section{B. Influence of QW states on the magnetic profile}

The sign and the magnitude of the deviation from the linear superposition $M_{\mathrm{QW}}(n)$ is directly related to the position of the QW states relative to the Fermi level. To see this connection we plot in Fig. 5 the trilayer moment profile and the deviation $M_{\mathrm{QW}}(n)$ together with the spectral density at $\bar{\Gamma}$ for spacer thicknesses $N$ from 15 to 18 atomic monolayers with FM coupling of the semi-infinite Fe crystals. If a mi- 

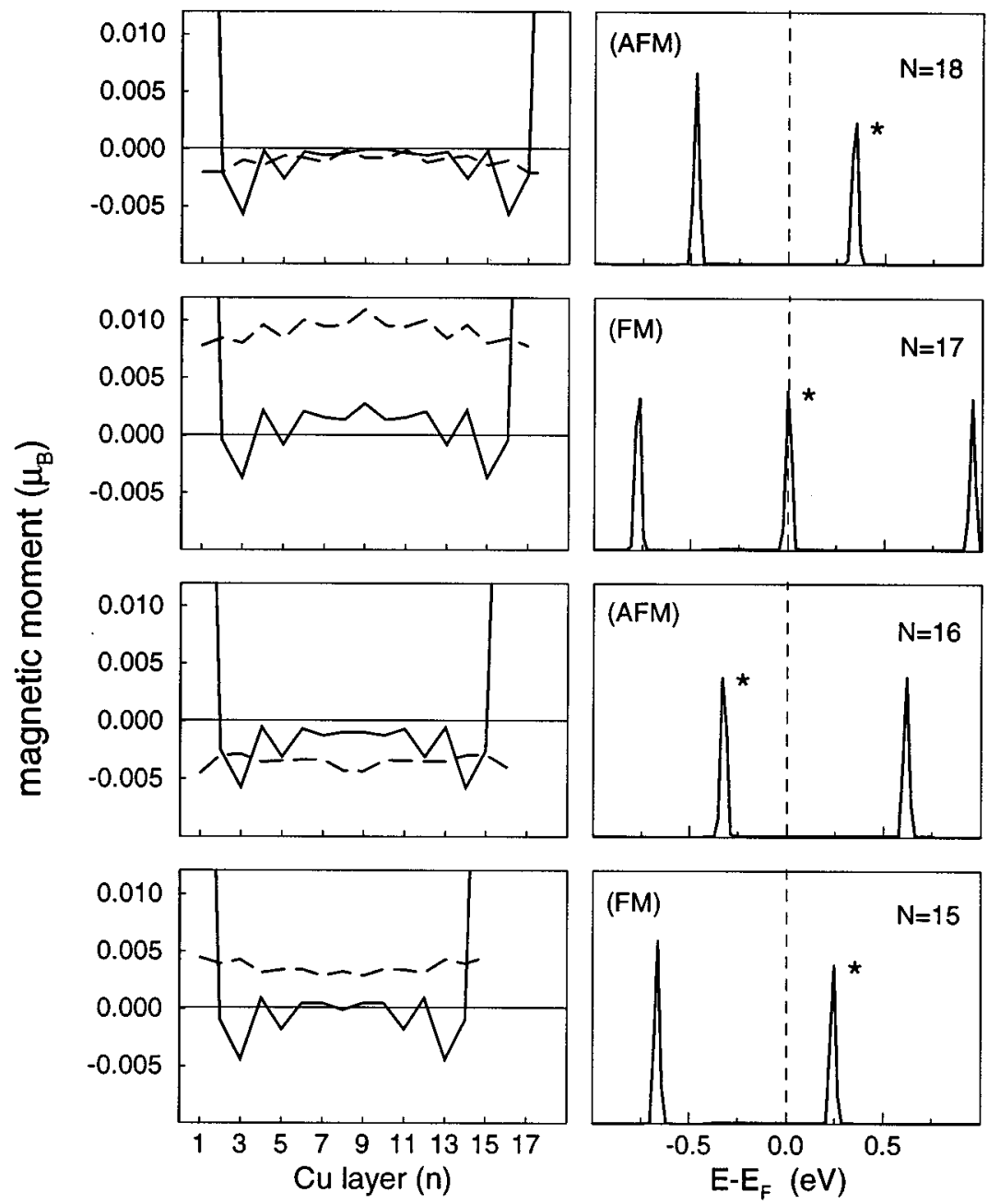

FIG. 5. In the left-hand panels we show the self-consistent moment profiles (solid lines) and $M_{\mathrm{QW}}(n)$ (dashed lines). The latter is the difference between the full calculation and the linear superposition, Eq. (12). In the right-hand panels we show the minority-spin spectral density at $\bar{\Gamma}$. The QW peaks closest to the Fermi level are marked by asterisks. The results corresponds to FM boundary conditions for $\mathrm{Fe} / \mathrm{Cu}_{N} / \mathrm{Fe}$ where $N$ varies from 18 to 15 as we proceed down the panels. The alignment of the moments, corresponding to the boundary conditions with the lowest total energy, is marked in parentheses in the right-hand panels. nority QW state is slightly below the Fermi level, e.g., for $N=16$, the occupation of the minority-spin channel is increased over that of the majority-spin channel and the QW contribution to magnetic moment together with $M_{\mathrm{QW}}(n)$ becomes negative. If, on the other hand, a QW state is slightly above the Fermi level, e.g., for $N=15, M_{\mathrm{QW}}(n)$ becomes positive. Finally, if a QW state is very close to the Fermi level as for $N=17, M_{\mathrm{QW}}(n)$ will be large while if the Fermi level falls between two QW states, $N=18, M_{\mathrm{QW}}(n)$ will be small. Therefore, the sign and magnitude of the deviation $M_{\mathrm{QW}}(n)$ between the complete moment profile and that obtained by the superposition may serve as a signature of the magnetic QW states around the Fermi level. The moment profile and therefore the total magnetic moment will consequently contain a constant shift due to this quantum-well effect. The shift will oscillate in sign and magnitude as the spacer thickness is increased. In experiments with a trilayer or a multilayer device it will be difficult to measure these characteristic shifts in the magnetic moment because the boundary conditions may alternate due to exchange coupling. However, if one puts the paramagnetic layers on top of the ferromagnetic crystal, i.e., replaces one of the ferromagnetic crystals by a vacuum, the boundary condition will remain fixed. The quantum well will now be formed between the ferromagnetic crystal and vacuum. QW states crossing the Fermi energy, as the number of paramagnetc coverlayers is increased, will now give rise to constant shifts in the magnetic profile of the paramagnet, positive or negative. Indications of such magnetic effects were seen by Wieringa et al. ${ }^{23}$ Strong oscillations in the magnetization induced second harmonic generation (MSHG) signal were found for a $\mathrm{Co} / \mathrm{Cu}$ system as a function of the thickness of the $\mathrm{Cu}$ coverlayer. Recent calculations by Mirbt et al. indicate dramatic changes in the magnetic beahaviour of Pd on top of $\mathrm{Ag}$ as the number of Pd layers was increased due to this QW effect. ${ }^{24}$

\section{Exchange coupling}

The shape of the deviation from the linear superposition, $M_{\mathrm{QW}}(n) \mathrm{Eq}$. (12), is of conceptual importance for the exchange coupling. In the Dyson series in Eq. (1) the second and the third terms $\Delta G_{L}^{\sigma}$ and $\Delta G_{R}^{\sigma}$ give the main contribution to the magnetic profile in the trilayer. On the other hand, the mixed interaction term $\Delta G_{\mathrm{QW}}^{\sigma}$, which is directly related to the QW states, is generally considered to give rise to the main contribution to the magnetic exchange coupling between the two Fe crystals. ${ }^{8,25,26}$ However, the QW states give an almost constant, positive or negative, contribution to the magnetization. Hence, we realize that the exchange coupling cannot be regarded as a transfer of an oscillating magnetic moment, but is rather caused by the energy difference due to QW states. 
By comparing the total energy for the trilayer with antiferromagnetic and ferromagnetic boundary conditions we find the alignment of the ferromagnetic moments. ${ }^{27}$ The coupling, ferromagnetic (FM) or antiferromagnetic (AFM), is marked within parentheses in Fig. 5. The obtained coupling is in good agreement with experiments. ${ }^{28}$

\section{CONCLUSION}

We have shown that the magnetic moments in a magnetic trilayer to a first approximation may be described as a linear superposition of two independent magnetic Friedel tails from two single freestanding interfaces. The small and almost layer-independent deviation from this simple superposition is shown to be a direct consequence of the existence of $\mathrm{QW}$ states close to the Fermi level. The fact that the superposition gives a good description of the magnetic moments makes it possible via a recursion relation to predict the magnetic profile for one particular interlayer thickness if it is known for two other thicknesses. The validity of the superposition has been shown for $\mathrm{Fe} / \mathrm{Cu}_{N} / \mathrm{Fe}$ trilayers only but should apply to other ferromagnetic/paramagnetic systems as well. However, the simple interpretation of the deviation in magnetic moment does not hold if there are no well-defined QW states close to the Fermi level. In this respect the $\mathrm{Fe} / \mathrm{Cu}_{N} / \mathrm{Fe}$ trilayer system is ideal but not unique and the QW effects on the magnetic moment presented in this work may be applied to other systems.

\section{ACKNOWLEDGMENTS}

We would like to thank Magnus Aldén, Lars Nordström, and Igor Abrikosov for numerous discussions and helpful comments. The Center for Atomic-scale Materials Physics is sponsored by the Danish National Research Foundation. The Uppsala group is grateful to the Swedish Natural Science Research Council for financial support. This work forms part of a project supported by a European Community SCIENCE contract.
${ }^{1}$ P. Grünberg, R. Schreiber, Y. Pang, M.B. Brodsky, and H. Sowers, Phys. Rev. Lett. 57, 2442 (1986).

${ }^{2}$ S.S.P. Parkin, N. Moore, and K.P. Roche, Phys. Rev. Lett. 64, 2304 (1990).

${ }^{3}$ S.S.P. Parkin, R. Bhadra, and K.P. Roche, Phys. Rev. Lett. 66, 2152 (1991).

${ }^{4}$ M.N. Baibich, J.M. Broto, A. Fert, F. Nguyen van Dau, F. Petroff, P. Etienne, G. Creuzet, A. Friederich, and J. Chazelas, Phys. Rev. Lett. 61, 2472 (1988).

${ }^{5}$ G. Binasch, P. Grünberg, F. Saurenbach, and W. Zinn, Phys. Rev. 39, 4828 (1989).

${ }^{6}$ J.E. Ortega and F.J. Himpsel, Phys. Rev. Lett. 69, 844 (1992).

${ }^{7}$ L. Nordström, P. Lang, R. Zeller, and P.H. Dederichs, Europhys. Lett. 29, 395 (1990).

${ }^{8}$ P. Bruno, Phys. Rev. B 52, 411 (1995).

${ }^{9}$ H.L. Skriver and N.M. Rosengaard, Phys. Rev. B 43, 9538 (1991)

${ }^{10}$ O.K. Andersen, Phys. Rev. B 12, 3060 (1975).

${ }^{11}$ H.L. Skriver, The LMTO Method (Springer-Verlag, Berlin, 1984).

${ }^{12}$ O.K. Andersen and O. Jepsen, Phys. Rev. Lett. 53, 2571 (1984).

${ }^{13}$ O.K. Andersen, O. Jepsen, and D. Glötzel, in Highlights of Condensed-Matter Theory, edited by F. Bassani, F. Fumi, and M.P. Tosi (North-Holland, New York, 1985).

${ }^{14}$ O.K. Andersen, Z. Pawlowska, and O. Jepsen, Phys. Rev. B 34, 5253 (1986).
${ }^{15}$ S.H. Vosko, L. Wilk, and M. Nusair, Can. J. Phys. 58, 1200 (1908).

${ }^{16}$ B. Wenzien, J. Kudrnovsky, V. Drchal, and M. Sob, J. Phys. Condens. Matter 1, 9893 (1989).

${ }^{17}$ M. Aldén, H.L. Skriver, S. Mirbt, and B. Johansson, Phys. Rev. Lett. 69, 2296 (1992).

${ }^{18}$ M. Aldén, S. Mirbt, H.L. Skriver, N.M. Rosengaard, and B. Johansson, Phys. Rev. B 46, 6303 (1992).

${ }^{19}$ M. Aldén, H.L. Skriver, S. Mirbt, and B. Johansson, Surf. Sci. 315, 157 (1994).

${ }^{20}$ S. Mirbt, H.L. Skriver, M. Aldén, and B. Johansson, Solid State Commun. 88, 331 (1993).

${ }^{21}$ J. Kudrnovsky (private communication).

${ }^{22}$ J. Mathon, M. Villerer, and D.M. Edwards, J. Phys. Condens. Matter 4, 9873 (1992).

${ }^{23}$ H.A. Wierenga, W. de Jong, M.W.J. Prins, Th. Rasing, R. Vollmer, A. Kirilyuk, H. Schwabe, and J. Kirschner, Phys. Rev. Lett. 8, 1462 (1995).

${ }^{24}$ S. Mirbt, H.L. Skriver, and B. Johansson (unpublished).

${ }^{25}$ P. Bruno, J. Magn. Magn. Mater. 121, 248 (1993).

${ }^{26}$ P. Lang, L. Nordström, R. Zeller, and P.H. Dederichs, Phys. Rev. Lett. 12, 1927 (1993)

${ }^{27}$ A.M.N. Niklasson, S. Mirbt, M. Aldén, H.L. Skriver, and B. Johansson, J. Magn. Magn. Mater. 148 (1995).

${ }^{28}$ M.T. Johnson, S.T. Purcell, N.W.E. McGee, R. Coehoorn, J. aan de Stegge, and W. Hoving, Phys. Rev. Lett. 68, 2688 (1992). 